\title{
An Experimental Study of the Microstructures and Mechanical Properties of Swine Cruciate Ligaments*
}

\begin{abstract}
Shunji HIROKAWA** and Tsutomu SAKOSHITA**
Tensile tests were performed on bone-ligament-bone (BLB) units, sections of ligament, and individual collagen fascicles all from the knees of swine hind legs. A universal testing machine was used for the tensile tests of the BLB units. A specially designed test apparatus was used for the tensile tests of ligament sections and fascicles. The strain values were calculated from the elongation values recorded by a video camera. The results showed that the BLB's stiffness was greatest, followed by the fascicles and the ligament sections. The results are contrary to the popular notion that because the ligament is composed of collagen fascicles in a matrix whose stiffness is almost negligible, the ligament should not be stiffer than the fascicles that compose it. The stiffness might have been caused by mechano-chemical interactions between fascicles and matrix, or contributions from the membranous septum that combines fascicles.
\end{abstract}

Key Words : Cruciate Ligament, Structure-Property Relationship, Collagen Fascicles, Ligament Section, Bone-Ligament-Bone Unit, Stiffness, Location Dependence, Microstructure

\section{Introduction}

Knowledge of the knee ligaments' mechanical properties is essential for providing correct surgical treatment or replacement in the event of rupture. Studies on the mechanical properties of ligaments are extensive ${ }^{(1)-(4)}$. Most of these studies concern only macro-structural criteria. However, the cruciate ligaments' mechanical properties are known to vary from area to area within the ligament, depending what part of the tensile load each part shares during knee motion. Studies on the cruciate ligaments' mechanical properties and their variation from area to area have presented widely varied results depending upon the specimen and the experimental conditions. The ligament is composed of fascicle bundles, each consists of a number of collagen fascicles. The fascicles are made up of fibers and fibrils. To gain insight into the mechanisms of ligament rupture, it may be helpful to elucidate the basic relationships between the microstructures and their mechanical

* Received 14th June, 2002 (No. 02-4106)

** Department of Intelligent Machinery and Systems, Faculty of Engineering, Kyushu University, 4-2-1 Ropponmatsu, Chuo-ku, Fukuoka 810-8560, Japan. E-mail : shunhiro@rc.kyushu-u.ac.jp properties in the ligaments ${ }^{(5)}$.

In this study, we investigated the relationships between the structures and their mechanical properties. We used bone-ligament-bone (BLB) units, sections of ligament, and individual collagen fascicles, all obtained from the knees of swine hind legs. Tensile tests were performed on each and the results were compared with each other and with those in the literature. Furthermore, microscopic observations were carried out on three orthogonal sections obtained from various parts of ligament respectively, in order to investigate the collagen fascicles' orientations and distributions, thereby obtaining basic knowledge of the relationship between the microstructures and their mechanical properties.

\section{Materials and Methods}

\section{1 Materials}

The following three kinds of test subject were obtained from the fresh knees of swine hind legs, which were harvested from the butchery.

2.1.1 Bone-Ligament-Bone (BLB) units To prepare the BLB units, the distal one-third of the femur and proximal one-third of the tibia were used, and all soft tissues were removed from the knee, leaving the ligament intact. We cut a slit into the 
femoral bone along its longitudinal axis, and inserted a square metal plate with holes at the both ends. Then a hole was drilled through the bone, and a bolt was inserted through both the bone and the metal plate. The same was done for the tibia. We removed the medial half of the femoral condyle from one unit to obtain an unobstructed view of the Anterior Cruciate Ligament (ACL) (Fig. 1) and the lateral half of the condyle from another unit to obtain a clean view of the Posterior Cruciate Ligament (PCL). We attached several markers to the ACL and PCL ligament surfaces to measure strain during the tests. As soon as preparations were completed, both the ends of the above mentioned metal plates were hooked to the test apparatus and the tensile tests were performed.

\subsubsection{Ligament sections A ligament was} resected from the bones, laid in the shallow groove of an acrylic block and frozen in a freezer. The part of the ligament projecting from the block was planed using a microtome blade to obtain a section of tissue about $0.5 \mathrm{~mm}$ in thickness. Figure $2(\mathrm{a})$ and (b) show views of the process and a section respectively. The resulting section was trimmed to form a rectangle about $30 \mathrm{~mm}$ long and $15 \mathrm{~mm}$ wide. The same method was used to slice an ACL ligament from the anterior side to the posterior side in parallel as shown in Fig. 3, to obtain about five sections. All sections

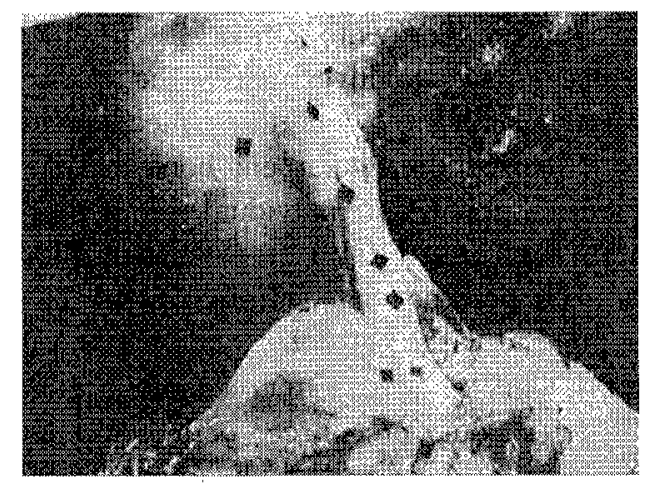

Fig. 1 A photograph of bone-ligament-bone unit
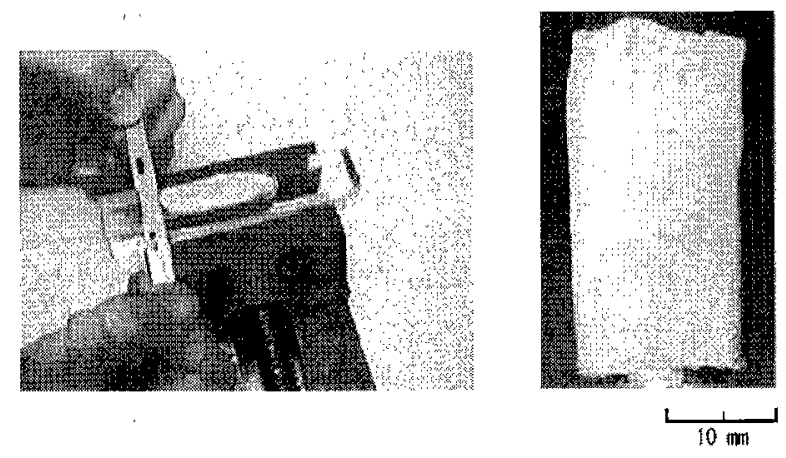

(a) Method of preparing a ligament (b) A ligament section

Fig. 2 Preparation of a ligament section were double-wrapped with gauze and aluminum foil and placed in a freezer until use.

2.1.3 Collagen fascicles Figure 4 illustrates the stages in extracting a single fascicle without excessive pulling. The ligament was first divided into four parts along the longitudinal axis. A fascicles' bundle was resected from one of the quartered ligaments, and a single fascicle was gently peeled from the quartered ligament using tweezers. The size and shape of a single fascicle were measured under a photomicroscope. Since the cross section of the fascicle was reported to be almost circular ${ }^{(5)}$, we calculated the cross sectional area from its diameter. All specimens peeled from any of the quartered ligaments were put in a bath filled with saline solution and the bath was placed in a freezer until use:

\section{2 Methods}

2.2.1 Tensile tests of BLB units The tensile tests of both ACL and PCL BLB units were done on a universal testing machine. On the assumption that the cross-section of a ligament is an ellipse in shape, we calculated the cross sectional area from a set of diameters recorded from the frontal and lateral/ medial directions by, video camera. The ligament's length was divided into three equal intervals, and measurements were done at each dividing line. The areas of the three sections were calculated and then averaged. Stress values were calculated by dividing the load measurement by the value of the cross ${ }^{-}$sec-

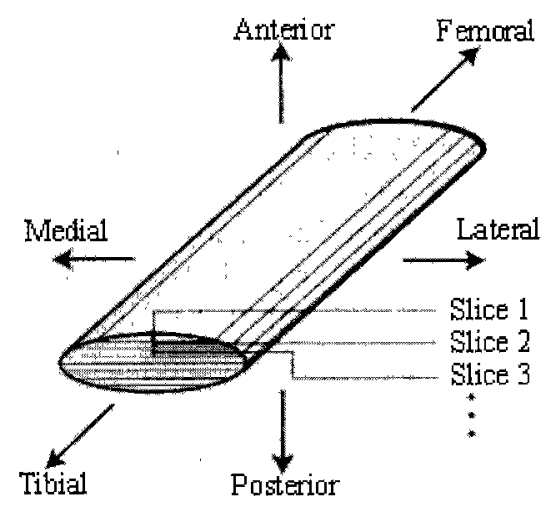

Fig. 3 Preparation of ligament sections by slicing a ligament in parallel to the sagittal plane

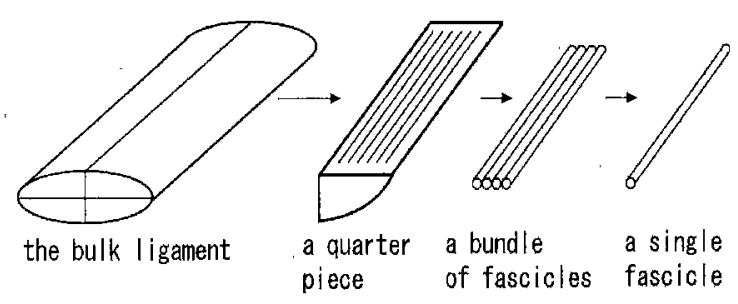

Fig. 4 Stages in extracting a single fascicle from a ligament 
tional area. The positions of markers during each test were recorded by a video camera. A pair of markers attached to the same fascicle was used to calculate strain. The same was done for all other pairs of markers, and all strain values thus obtained were averaged, thereby decreasing the effects on the results caused by for example, stress transfer between the bone and ligament or parallax of the camera. Since large scatter was expected to the results from the biological material, we applied the statistical analysis (T-test) to the results. The same was done for the following ligament sections and fascicles.

2.2.2 Tensile tests of ligament sections and fascicles A specially designed test apparatus was used to perform the tensile tests on ligament sections and fascicles (Fig. 5). Two threaded loading arms attached to an actuator in such a way that it pulled a specimen in opposite directions simultaneously. The actuator was a stepper motor (CSK226-AP, Oriental Motor Co., Japan) and a load cell (LTS-50KA rated at $50 \mathrm{kgf}(490 \mathrm{~N})$, or LTS-2KA rated at $20 \mathrm{kgf}$ (196 N), Kyowa Dengyo Co., Japan). The actuator was controlled by a personal computer (PC-9801DS, NEC Co., Japan) through the $\mathrm{I} / \mathrm{O}$ and $\mathrm{A} / \mathrm{D}$ boards and digital indicator (F340, Unipulse Co., Japan). The apparatus can apply a pulling force of $0-50 \mathrm{kgf}(0-$ $490 \mathrm{~N})$ with an accuracy of $0.001 \mathrm{kgf}(0.0098 \mathrm{~N})$, and can change pulling speed from $0-20 \mathrm{~mm} / \mathrm{s}$. A video camera (DCR-TRV900, Sony Co., Japan) was positioned $300 \mathrm{~mm}$ above the specimen to record the specimen's motion and deformation. For tensile tests of the ligament sections, a load cell rated at $50 \mathrm{kgf}$ $(490 \mathrm{~N})$ was used. Each specimen was thawed to room temperature before testing. As shown in Fig. 6 (a), each end of a specimen was held between two stainless plates whose opposing surfaces had been lined with waterproof sandpaper. The larger stainless plate was connected to the loading arm. An instantaneous adhesive agent whose principal ingredient is cyan acrylic ester monomer was applied to the sand-

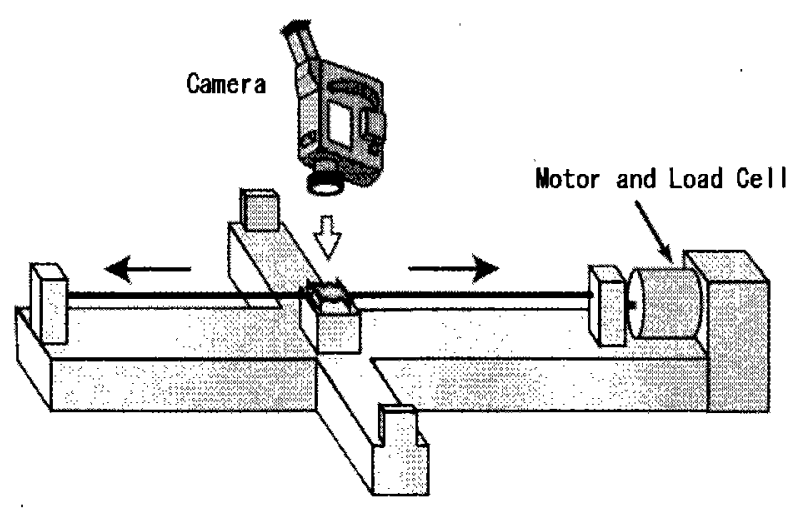

Fig. 5 Schematic drawing of the tensile test apparatus paper. The smaller stainless plate was fastened to the larger one with screws to secure the specimen to the sandpaper. Care was taken not to fasten the plates too tightly to squeeze the specimen. The apparatus was activated, and the specimen was pulled at rate of $0.33 \mathrm{~mm} / \mathrm{s}^{(4)}$ until it ruptured. As the cross section of the specimen was almost square in shape, the cross sectional area was calculated from its width and thickness as recorded by video camera. Strain was calculated from elongation between both the ends recorded by video camera.

For tensile tests of the collagen fascicle, the same apparatus was used, but a load cell rated at $20 \mathrm{kgf}$ (196 N) was substituted. After being thawed to room temperature, each end of the fascicle was fixed to a stainless plate by two sheets of sandpaper and an adhesive agent Fig. 6 (b). Since collagen fascicles dry quickly at room temperature, the specimen was kept in saline solution during testing. The specimen was pulled at rate of $0.10 \mathrm{~mm} / \mathrm{s}$ until it ruptured. Strain was calculated the same way as it was for the ligament sections. Stress was calculated by dividing tensile force by the value of the previously obtained cross sectional area.

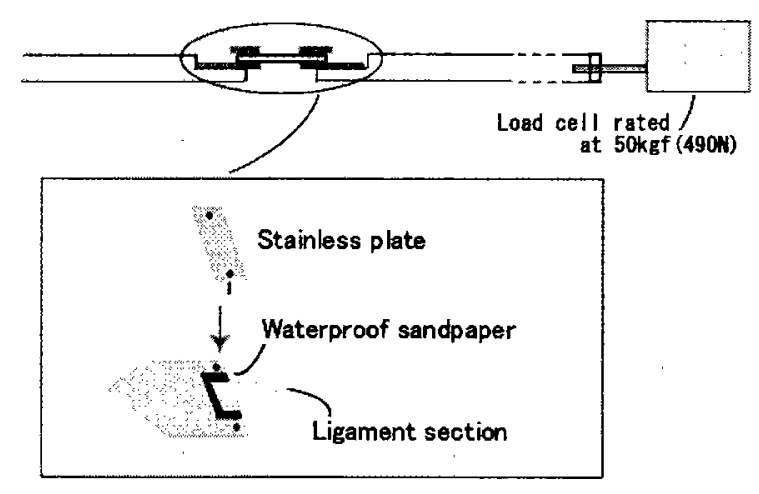

(a) A ligament section

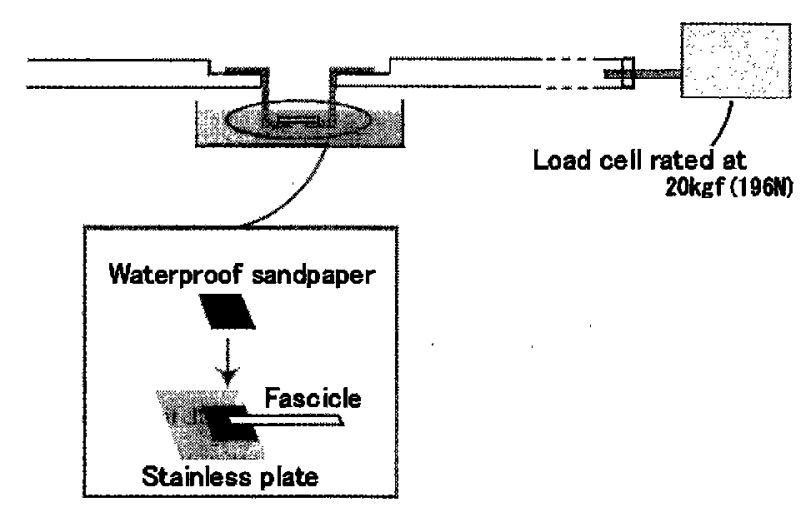

(b) A single fascicle

Fig. 6 Method used to clamp a specimen to the loading arms 


\section{3 Microscopic observation}

After excision, the ACL and PCL specimens were sectioned in parallel to the sagittal, frontal or coronal plane. The sections were fixed in $10 \%$ neutral buffered formalin for $48 \mathrm{~h}$ at $4^{\circ} \mathrm{C}$, decalcified in $10 \%$ EDTA (pH 7.4), and embedded in paraffin. Each section was stained with Masson trichrome to highlight the collagen fascicles exclusively. Twenty $5 \mu \mathrm{m}$ thick slices were made. Eight coronal sections were prepared by dividing the ligament's length at equal intervals between the femoral and tibial insertions. Three frontal sections were prepared by dividing the ligament's thickness at equal intervals from anterior to posterior. Two slides were made from each frontal section for a total of six. Three sagittal sections were made by equally dividing the ligament's width. Two slides were made from each section for a total of six. Using the micrographs from twenty slides, we measured orientation and distribution of the collagen fascicles in the ligaments and the size and morphology of a single fascicle.

\section{Results}

\subsection{Tensile tests}

Figures 7 to 10 give the results of the tensile tests. Among the ACL sections, significant location-dependent variations in mechanical properties were found, depending upon the site from which the section was sliced. Figure 7 shows the results obtained from the ACL sections. In the figure, mechanical properties are arranged in terms of tangent modulus, tensile strength, and strain at failure. The tangent modulus was introduced from the tangent at $8-12 \%$ of strain, that is, at the linear region of the stress-strain curve. Since the results clearly demonstrated location dependent characteristics, we grouped the results with respect to three locations: the outermost layer of the anterior side, the remainder of the anterior side and the posterior side. The values of tangent modulus of

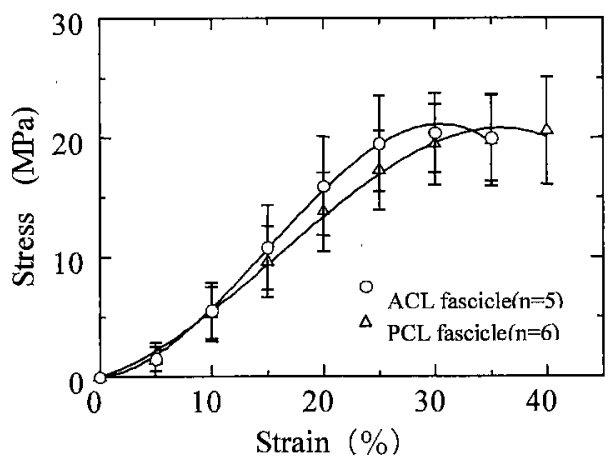

(a) Fascicles the outermost layer of the anterior side, the remainder of the anterior side and the posterior side were respectively $108.8 \pm 15.7 \mathrm{MPa}, 67.5 \pm 5.1 \mathrm{MPa}$ and $22.1 \pm 6.7$ MPa. The values of tensile strength of the outermost layer, the anterior side and the posterior side were respectively $19.8 \pm 5.6 \mathrm{MPa}, 7.6 \pm 3.1 \mathrm{MPa}$ and $6.8 \pm 2.2$ $\mathrm{MPa}$. The values of strain at failure of the outermost, the anterior and the posterior were respectively $22 \pm$ $7.1 \%, 15 \pm 7.3 \%$ and $33 \pm 5.3 \%$. Both tangent modulus and tensile strength decreased from the outermost
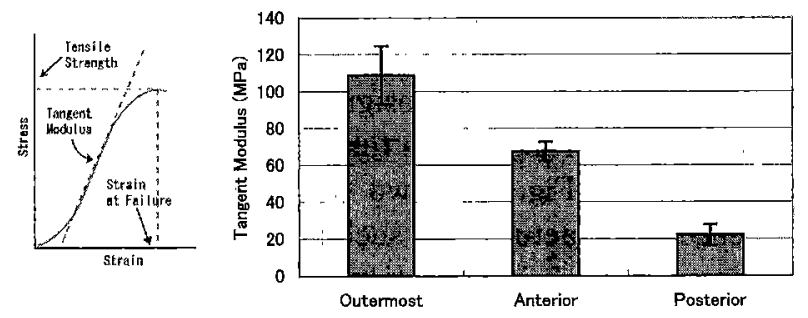

(a) Tangent modulus

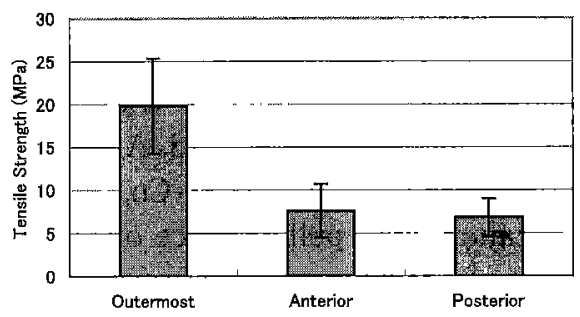

(b) Tensile strength

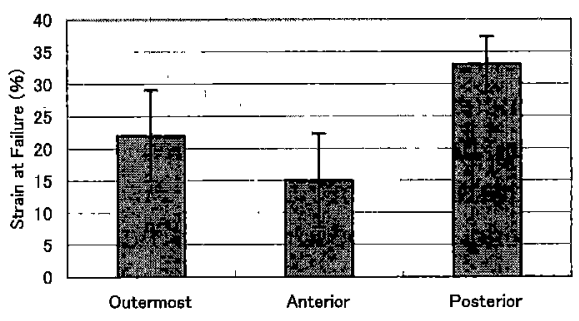

(c) Strain at failure

Fig. 7 Mechanical properties of each ACL section group

Fig. 8 Stress-strain characteristics of the ACL vs. PCL 


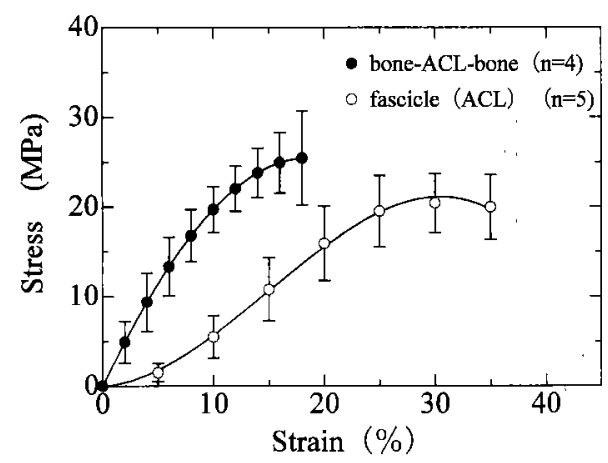

(a) $\mathrm{ACL}$

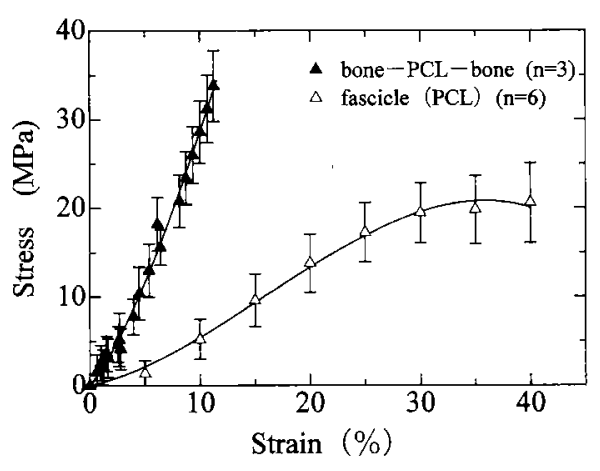

(b) PCL

Fig. 9 Stress-strain characteristics of the BLB units vs. fascicles

layer to the posterior side. As is shown in Figs. 8 - 10, a specimen whose tangent modulus is higher than that of other specimens had a corresponding lower strain at failure. However, the outermost layer of the anterior not only had a higher tangent modulus but also a higher strain value at failure than did the other two groups. This suggests that the histology as well as the properties of the outermost layer is special.

Among the PCL sections, we did not find such significant location-dependent variations in mechanical properties as the ACL sections. This may be partially because the fascicles in the PCL align in parallel like tendons.

Figure $8(a)$ and (b) show the stress ${ }^{-}$strain curves of the ACL and PCL fascicles and the bone$\mathrm{ACL}-$ bone and bone-PCL-bone units respectively. In Fig. 8(a), the difference between the two curves is not significant. Thus we may conclude that the mechanical properties of the ACL and PCL fascicles are almost equivalent. In Fig. 8(b), the tangent modulus of the PCL is significantly higher than that of the ACL. The rupture mode was a tibial avulsion for the ACL and a femoral avulsion for the PCL.

Figure $9(\mathrm{a})$ and (b) show the stress-strain curves of fascicles and BLB units for the ACL and PCL respectively. They are a rearrangement from Fig. 8 done to compare the properties of the fascicles and BLB units. The figures show that both BLB units had a higher tangent modulus and lower strain at failure than collagen fascicles. This suggests that a BLB unit is stiffer than an individual fascicle.

We arranged the results from Figs. 8 to 9 in terms of tangent modulus, tensile strength and strain at failure as shown in Fig. 10. The values of tangent modulus of the ACL fascicles, the PCL fascicles, the bone-ACL-bone units and the bone-PCL-bone units were respectively $104.2 \pm 20.4 \mathrm{MPa}, 85.6 \pm 11.6 \mathrm{MPa}$, $204.5 \pm 29.9 \mathrm{MPa}$ and $312.8 \pm 35.0 \mathrm{MPa}$. "The tangent modulus of each specimen was introduced from the

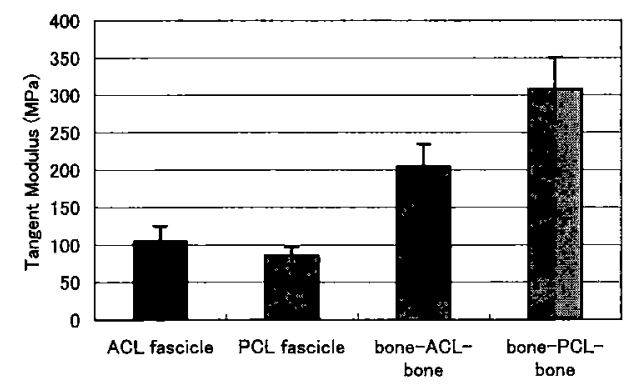

(a) Tangent modulus

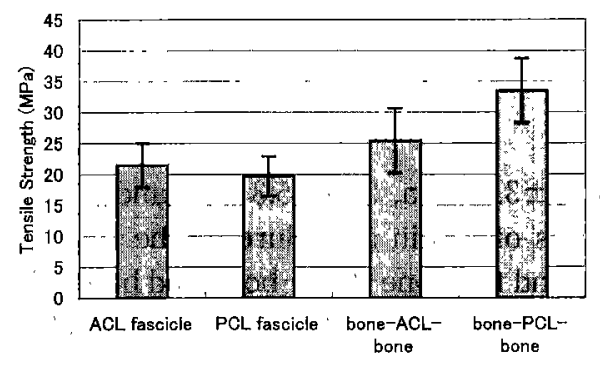

(b) Tensile strength

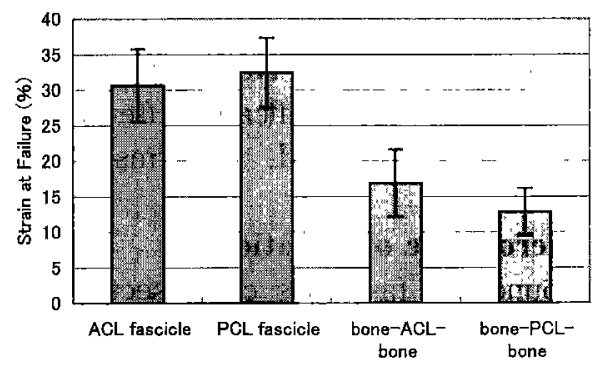

(c) Strain at failure

Fig. 10 Mechanical properties of ACL and PCL fascicles and BLB units respectively

tangent: for the ACL and PCL fascicles at $10-20 \%$ strain of the stress-strain curve; for the bone-PCLbone units at $2-6 \%$; and for the bone-ACL-bone units at $4-8 \%$. The value of tensile strength of the ACL and PCL fascicles and the bone-ACL-bone and bone-PCL-bone units were respectively $21.5 \pm 3.6$ 


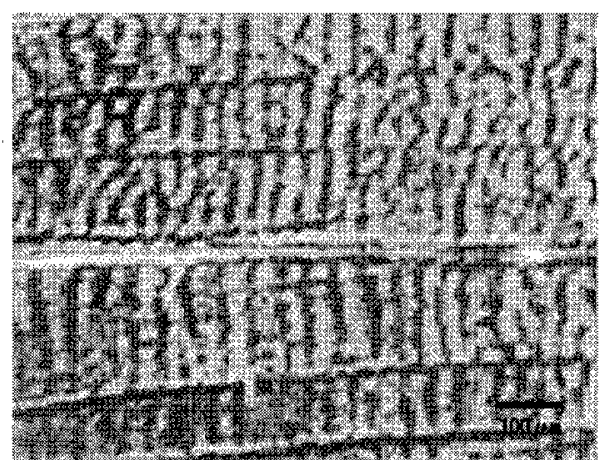

(a) Sagittal section

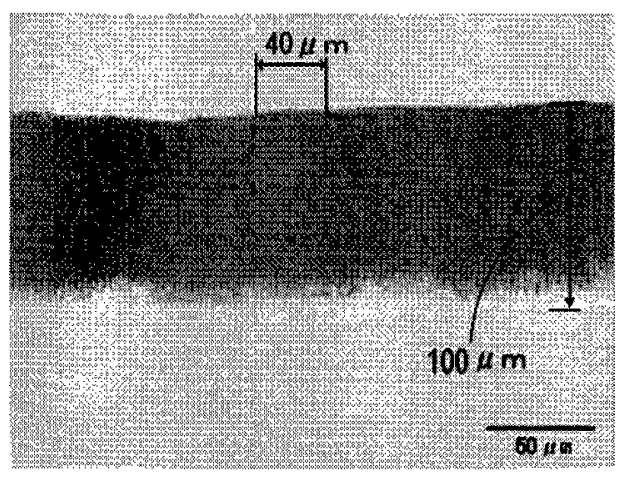

(b) A single fascicle

Fig. 11 Micrographs of the ACL

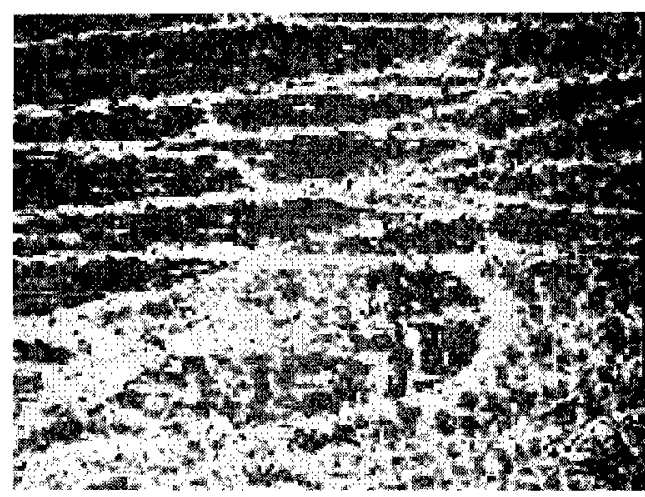

(a) ACL

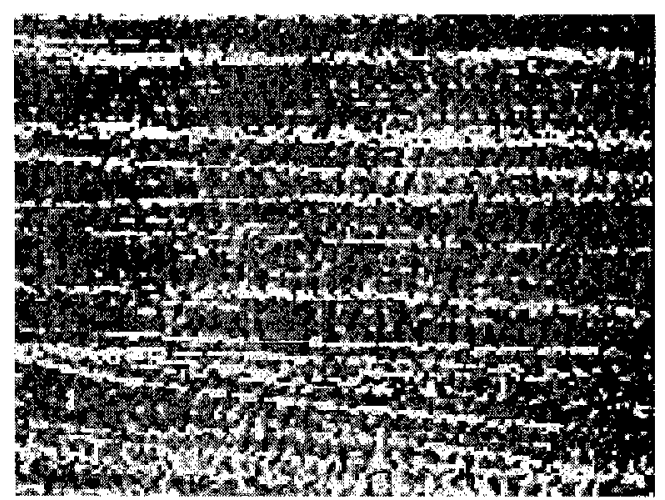

(b) PCL

Fig. 12 Typical micrographs of the ligament's midsubstance sagittal section

$\mathrm{MPa}, 19.7 \pm 3.2 \mathrm{MPa}, 25.4 \pm 5.3 \mathrm{MP}$, and $33.8 \pm 4.2 \mathrm{MP}$. The values of strain at failure of the $\mathrm{ACL}$ and $\mathrm{PCL}$ fascicles and the bone-ACL-bone and bone-PCL-bone units were respectively $30.7 \pm 5.1 \%, 32.4 \pm 4.9 \%, 16.9 \pm$ $4.7 \%$ and $11.2 \pm 2.5 \%$. When the $\mathrm{ACL}$ and PCL are part of an actual joint (as in the BLB units), the PCL has higher tangent modulus and lower strain at failure than the ACL $(p<0.005)$. However, at the fascicle level, no statistically significant differences appear between the ACL and PCL in terms of the three parameters.

\section{2 Microscopic observation}

We found the fascicle's cross-section is almost circular as reported in the literature ${ }^{(5)}$, and therefore we calculated the cross ${ }^{-}$sectional area of a fascicle from its diameter. Microscopic observation of the twenty slides revealed that the PCL fascicles run longitudinally in parallel. The mean diameter and length of both ACL and PCL fascicles were about the same: approximately $100 \mu \mathrm{m}$ in diameter and $20 \mathrm{~mm}$ long. The ACL fascicles run almost in parallel in the mid-portion but turn posteriorly toward the tibial insertion. Figure 11 (a) is a sample micrograph of an ACL's sagittal section where fascicles are densely packed, and ( $b$ ) is a micrograph of a single fascicle.

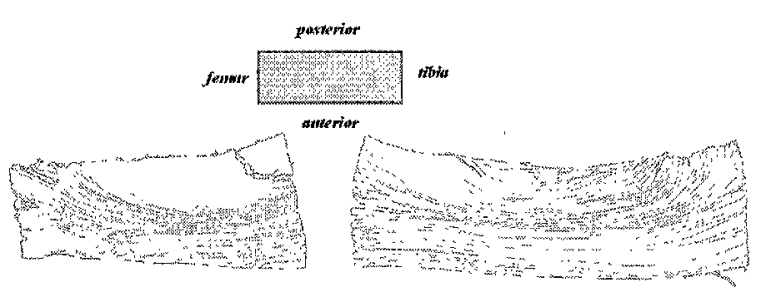

(a) $\mathrm{ACL}$

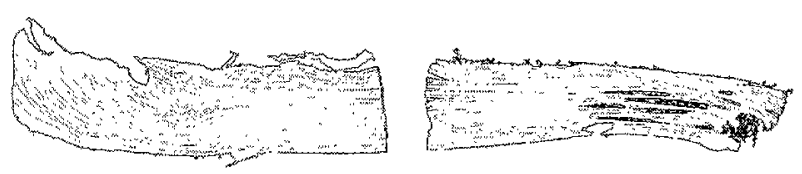

(b) PCL

Fig. 13 Binary code' images representing fascicles' arrangement in the ligament

Micrographs of the lateral view clearly showed that the ACL and PCL fascicles have uniform periodic undulations, or crimp patterns. Crimp patterns were observed along the longitudinal axis of the fascicle: the mean distance between crimp marks was approximately $40 \mu \mathrm{m}$. The value was identical to that of a single fascicle peeled from a fascicle bundle. Figure 12 ( a ) and (b) show typical micrographs obtained from the ACL's and PCL's midsubstance sagittal 


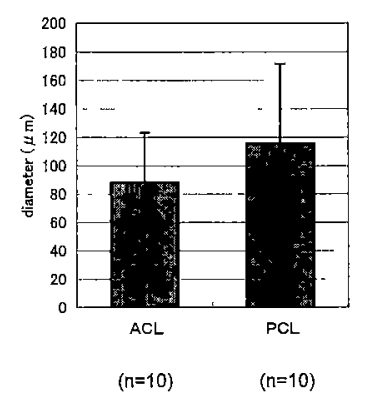

(a) Diameters

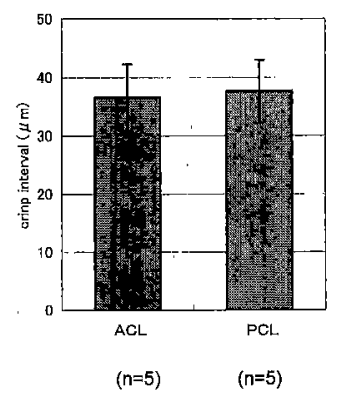

(b) Crimp intervals

Fig. 14 Morphology of the ACL and PCL fascicles

sections respectively. Also Figure $13(\mathrm{a})$ and (b) show binary code images representing fascicles' arrangement for the ACL and PCL respectively. The images were fabricated using conventional free software. The PCL fascicles are packed densely and uniformly in the entire area of the PCL. The ACL fascicles are packed densely and uniformly on the anterior side, whereas they are packed sparsely and irregularly on the posterior side. Figure 14 shows the mean and standard deviations of diameters and crimp marks for the ACL and PCL fascicles respectively. Although the mean diameter of a PCL fascicle appears larger than that of an ACL fascicle, the difference is not significant because of large statistical variations. We conclude that the morphology and mechanical properties of the ACL and PCL fascicles do not differ significantly.

\section{Discussion}

\subsection{Differences in microstructure and property between the ACL and PCL}

The fact that the bone-PCL-bone unit's tangent modulus was higher than that of the bone-ACL-bone unit's [Fig. $9(\mathrm{a})$ ] is in agreement with previous studies done on human ACLs ${ }^{(6)}$ and rabbits $\mathrm{ACLs}^{(7)}$. However, the ACL and PCL fascicles did not differ significantly in size or morphology (Fig. 8, Fig. 12). By dry weight, the human ACL contains $83 \%$ collagen and the PCL $90 \%{ }^{(8)}$. Microscopic observation revealed that the PCL fascicles are packed densely and uniformly in every portion of the ligament, while the ACL fascicles are aligned sparsely and irregularly on the posterior side and have a tendency to turn posteriorly toward the tibial insertion. The tensile test results and microscopic observation suggest that the difference in mechanical properties between the ACL and PCL ligaments is caused by the differences in the fascicles' density and orientation.

\subsection{Location dependence of mechanical prop- erties in the ACL}

The ACL is anatomically divided into three

groups : the anterior-medial, anterior-lateral and posterior groups. However, experiments on rabbit $\mathrm{ACLs}^{(1)}$ and on a human ACL Butler et al. ${ }^{(2)}$ have revealed no differences in mechanical properties between the anterior-medial and anterior-lateral groups. Accordingly, we did not divide the anterior portion into medial and lateral groups for the tensile test. The results obtained from the swine ACL sections are in good agreement with those from Woo et al..$^{(1)}$ and Butler et al. ${ }^{(2)}$, indicating that the mechanical properties of the swine ACL section differed depending on the location. According to Yahia et al. ${ }^{(9)}$, the posterior band of the human ACL is composed of small fascicles packed sparsely in the posterior side and densely in the anterior, whereas the anterior band is formed of dense and thick collagenous fascicles. Microscopic observation of the swine ACL found that fascicles are packed sparsely in the posterior side and densely in the anterior. The outermost layer not only had a higher tangent modulus but also a higher strain at failure than did the anterior portion. Part of the cause might be that the portion of the outermost layer we observed was not damaged when the ligament was sliced. However, this fact alone cannot account for the degree of the difference. It is likely that the outermost section including the surface is different mechanically and histologically from the substance of the ACL. The outermost layer surrounding a bulk ligament is known variously as the epiligament, peritenon, or periligament ${ }^{(10)}$. This layer may play a role in shielding the ligament or providing nutrient to it. It should be also noted that the unusual relationship between the tangent modulus and strain at failure observed in the outermost layer was not seen clearly in the posterior ACL groups or in the PCL sections. Therefore we may conclude that the outermost layer has unique mechanical and histological characteristics.

\section{3 Structure-property relationship of ligament}

Collagenous tissue such as ligaments or tendons has a hierarchical structure composed of fascicle bundles, collagen fascicles, fibers, fibrils and so on ${ }^{(11)}$. In the literature about the microstructure of ligaments, explanations can be confusing because terms such as fibril and fiber are not used consistently. In this paper, we use the word fascicle to be synonymous with fiber bundle. We found that the BLB's tangent modulus is greater than that of the fascicles that reinforce the ligament (Fig. 9). The stress-strain curves for each kind of specimen from the ACL are shown in Fig. 15 ( a ) for comparison. Common sense suggests that because the ligament is composed of collagen fascicles in a matrix whose stiffness is almost negligible, the ligament should not be stiffer than the 


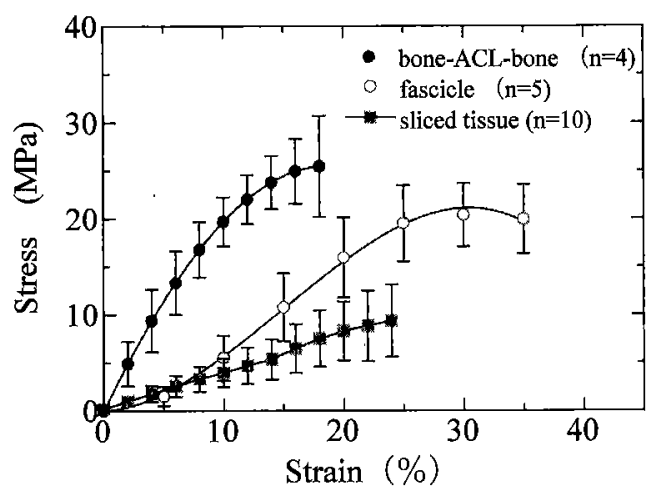

(a) Swain ACLs

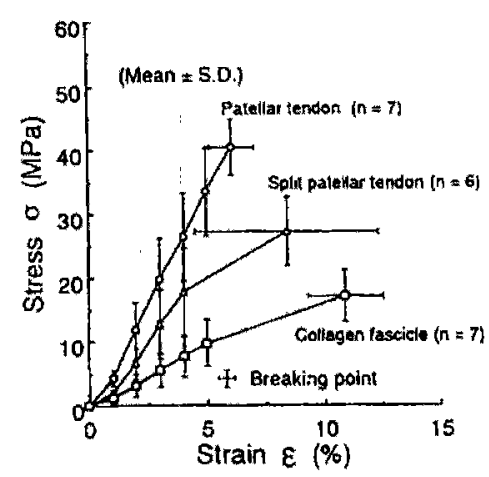

(b) Rabbit patellar tendons ${ }^{(13)}$

Fig. 15 Stress strain relationships for each structure in the tissue hierarchy

fascicles that compose it. It has been already verified experimentally that Young's modulus of fiber-reinforced plastic material falls between that of fiber and plastic base ${ }^{(14)}$. Nevertheless results similar to those in Fig. 15 (a) have been reported for rabbit tendon ${ }^{(13)}$ [Fig. 15(b)] and for rat-tail tendon ${ }^{(12)}$ as well. Figure 15 (a) also indicates that the tangent modulus of the ligament section is lowest among the three. This may be attributable to the fact that many fascicles were cut when the ligament was sliced. The results in Fig. 15 ( a ) and (b) may be caused by 1) mechano-chemical interactions between fascicles and between fascicles and their matrix, 2) contributions by the membranous septum (endoligament) to the tensile response of the ligament, or 3) visco-elastic resistance of an incompressive hyper-elastic matrix against the ligament's deformation. It is accepted that all fascicles in the tissue do not stretch equally. Thus, when a ligament is stretched, it is possible that-because of interactions between fascicles-the fascicles within the ligament may be stretched to a greater degree than the ligament itself. In Fig. 16, suppose the part of a fascicle in a triangular area is constrained to stretch because of the fascicles' interaction as shown in (b). More stress created in fascicle (2) than in fascicle (1), thereby creating more stress in the ligament (b) than that in (a). Even if this occurs only at a microscopic level, it could occur in numerous fibers in the ligament, resulting in an increase in the tangent modulus of the ligament. This hypothesis is based on the fact that the strain at failure of a BLB unit was significantly lower than that of a fascicle; the fascicles'strain at failure was $35 \%$ to $40 \%$, whereas that of the BLB units was only $10 \%$ to $15 \%$ as shown in Fig. 10.

To check how the above-mentioned endoligament affects the mechanical property of fascicles, we carried out a supplemental tensile test using a bundle of several fascicles, which were joined to each other by

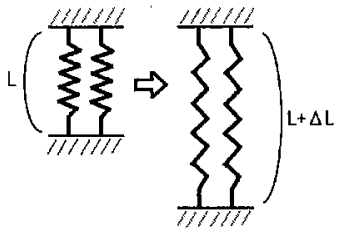

(a)

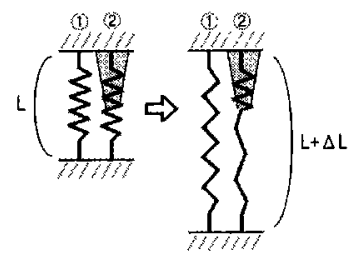

(b)
Fig. 16 Spring model to explain how the fascicles' mutual interaction increases the stiffness of tissue

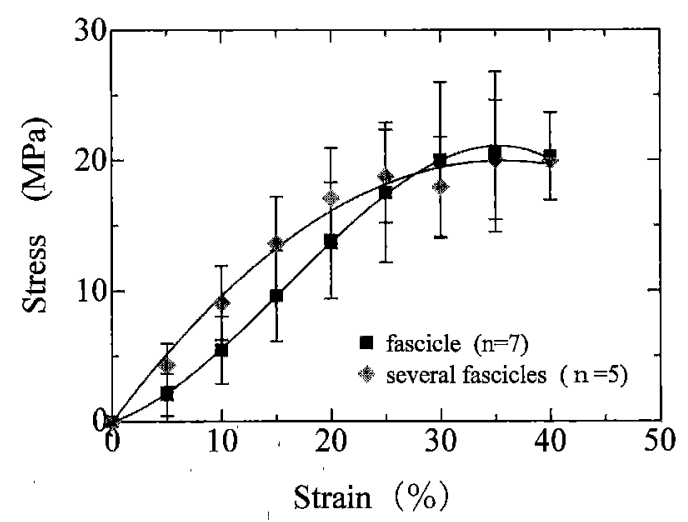

Fig. 17 Stress strain relationships of a single fascicle vs. a bundle of fascicles

the septum. We found that when fascicles are joined in a bundle; the bundle's tangent modulus becomes higher than that of a single fascicle, but not significantly higher (Fig. 17). This suggests the endoligament affects tensile response. Endoligament cells are reported to be basically the same size and shape as epiligament cells ${ }^{(10)}$. The epiligament is mechanically stronger than the individual fascicles. If the epiligament and endoligament have the same histology, then the endoligament may be also mechanically stronger than a fascicle. The endoligament not only combines fibrils into fascicles but also combines fascicles with each other. Peeling a single fascicle 
from a bundle for the tensile test might have damaged the endoligament layer and made the tangent modulus of the single fascicle lower. A hydrophilic matrix does not bear tensile force well, but does bear compressive force well because of the matrix incompressive nature. If within a ligament, the matrix constituents are sealed up in other structures, then the matrix could resist elongation. Hayashi et al. ${ }^{(15)}$ have reported that the mechanical response of a rabbit's patellar tendon is more typical of the matrix than of a fascicle. It is probable that the matrix plays a crucial role in the stress-strain characteristics of ligaments and tendons. Still the mechanism by which a matrix increases ligament stiffness is unknown.

\section{Conclusions}

To investigate the relationship between the microstructures in a ligament and its mechanical properties, tensile tests were performed on BLBs, ligament sections and collagen fascicles from the knees of swine hind legs. The stress-strain relationships among all three were compared with each other, and microscopic observations were made on fascicle orientation with the ligament as well as fascicles size and morphology. We found that the mechanical properties of the ligament varied depending the area of the ligament; stiffness decreased from the outermost part of the anterior section to the posterior section. In the PCL, mechanical properties did not vary depending on location. The tangent modulus was higher for the PCL than for the ACL. The location dependence of mechanical properties in the ACL, and the difference in mechanical properties between the ACL and PCL can be attributed to the orientation and distribution of the fascicles. There were no differences in mechanical properties among the fascicles in different part of the ACL, or between ACL and PCL fascicles. The BLB's tangent modulus was higher than that of the fascicles that reinforce the ligament.

This research program is supported by the Japanese Government Grand-in Aid for General Scientific Research (2002, No. 14580826).

\section{References}

(1) Woo, S.L.-Y., Newton, P.O., Mackenna, D.A. and Lyon, R.M., A Comparative Evaluation of the Mechanical Properties of the Rabbit Medial Collateral and Anterior Cruciate Ligaments, J. Biomechanics, Vol. 25, No. 4 (1992), pp. 377-386.

(2) Butler, D.L., Guan, Y., Kay, M.D., Cumming, J.F, Feder, S.M. and Levy, M.S., Location-Dependent Variations in the Material Properties of the Anterior Cruciate Ligament, J. Biomechanics, Vol. 25,
No. 5 (1992), pp. 511-518.

(3) Woo, S.L.-Y., Hollis, J.M., Roux, R.D., Gomez, M. A., Inoue, M., Kleiner, J.B. and Akeson, W.H., Effects of Knee Flexion on the Structural Properties of the Rabbit Femur-Anterior Cruciate Ligament-Tibia Complex (FATC), J. Biomechanics, Vol. 20, No. 6 (1987), pp. 557-563.

(4) Lyon, R.M., Woo, S.L.-Y., Hollis, J.M., Marcin, J. P. and Lee, E.B., A New Device to Measure the Structural Properties of the Femur-Anterior Cruciate Ligament-Tibia Complex, J. Biomechanical Eng., Vol. 111 (1989), pp. 350-354.

(5) Yamamoto, E., Hayashi, K. and Yamamoto, N., Mechanical Properties of Collagen Fascicles from the Rabbit Patellar Tendon, J. Biomechanical Eng., Vol. 121 (1999), pp. 124-131.

(6) Trent, P.S., Walker, P.S. and Wolf, B., Ligament Length Patterns, Strength and Rotational Axes of the Knee Joint, Clin. Orthop., Vol. 117 (1976), pp. 263-270.

( 7 ) Momersteeg, T.J.A., Blankevoort, L., Huiskes, R., Kooloos, J.G.M, Kauer, J.G.M. and Hendriks, J.C. M., The Knee Effect of Variable Relative Insertion Orientation of Human Knee Bone-LigamentBone Complexes on the Tensile Stiffness, J. Biomechanics, Vol. 28, No. 6 (1995), pp. 745-752.

(8) Fujii, K., Collagenous Metabolism in the Ligament, Seikeigeka Mook, (in Japanese), No. 58 (1989), pp. 16-22

(9) Yahia, L.H. and Drouin, G., Collagen Structure in Human Anterior Cruciate Ligament and Patellar Tendon, J. Mater. Sci., Vol. 23 (1988), pp. 37503755.

(10) Yahia, L.H. and Drouin, G., Microscopical Investigation of Canine Anterior Cruciate Ligament and Patellar Tendon: Collagen Fascicle Morphology and Architecture, J. Orthop. Research, Vol.7, No. 2 (1989), pp. 243-251.

(11) Kastelic, J., Galeski, A. and Baer, E., The Multicomposite Structure of Tendon, J. Connective Tissue Research., Vol. 6 (1978), pp. 11-23.

(12) Ikawa, E. and Yamamoto, N., Effects of Epitenon on the Mechanical Properties of Mouse Tail Tendon, Proceedings of the 12th JSME Autumn Bioengineering Conference and Seminar, (in Japanese), (2001), pp. 185-186.

(13) Hayashi, K., Miyazaki, H. and Hasegawa, Y., Mechanical Properties of Cells and Collagen Fiber, Proceedings of the 10th International Conference on Biomedical Engineering, (2000), pp. 55-56.

(14) Chemical Society of Japan, Ed., Fukugo Zairyo, (in Japanese), (1979), Gakkai Syuppan Center.

(15) Hayashi, K., Biomechanical Response of Tendon and Ligament, Proceedings of the 28th Annual Meeting of the Japanese Society for Clinical Biomechanics and Related Research, (in Japanese), (2001), p. 41. 